\title{
LED Driver With Self-Adaptive Drive Voltage
}

\author{
Yuequan $\mathrm{Hu}$ and Milan M. Jovanović, Fellow, IEEE
}

\begin{abstract}
This paper presents an LED driver circuit consisting of multiple linear current regulators and a voltage preregulator with adaptive output voltage. In the proposed driver, the output voltage of the preregulator is always self-adjusted so that the voltage across the linear current regulator of the LED string with the highest voltage drop is kept at the minimum value that is required to maintain the desired string current. Because the linear current regulators in this driver operate with the minimum voltages, the driver efficiency is maximized. The performance of the proposed driver was experimentally verified on a four-string LED setup with eight white LEDs in each string. The measured efficiency improvement of the linear current regulators was approximately $15 \%$ compared to the corresponding implementation with a constant preregulator voltage.
\end{abstract}

Index Terms-Adaptive drive voltage, LED driver, linear current regulator, sequential pulsewidth modulation (PWM) dimming.

\section{INTRODUCTION}

C URRENTLY, high-brightness LEDs are increasingly finding new applications in liquid crystal display (LCD) backlighting, automobiles, traffic lights, and general-purpose lighting, because of their superior longevity, low maintenance requirements, and improved luminance [1]-[3]. The brightness of LEDs is directly related to their current. An effective way to ensure that each LED produces similar light output is to connect them in series. However, a major drawback of a series connection of LEDs is their cumulative voltage drop that eventually limits the number of LEDs in a string. On the other hand, simple paralleling of LEDs or LED strings is not desirable because of current sharing problems related to both the LED's exponential voltage-current characteristic and the negative temperature coefficient of the LED's forward voltage drop [4].

There are several methods of driving multiple LED strings connected in parallel. A straightforward approach is to employ a current regulator for each string, as shown in Fig. 1. Generally, the current regulator can be of linear or switch-mode type. The approach employing linear regulators offers a low cost, but suffers from poor operating efficiency because the voltage drop across the linear regulator, i.e., the voltage difference between the input and output voltage of the linear regulator, cannot be minimized under all operating conditions. Namely, the input voltage of the linear current regulators needs to be set based on the worst-case condition, i.e., for the maximum LED string voltage drop, to ensure that the current of each LED string is regulated in the entire temperature and current range. As a result, the linear regulators driving LED strings that have

Manuscript received March 13, 2008; revised June 20, 2008. Current version published December 9, 2008. This paper was presented at the Applied Power Electronics Conference (APEC), February 24-28, 2008, Austin, TX, USA. Recommended for publication by Associate Editor M. Ponce-Silva.

The authors are with Delta Power Electronics Laboratory, Delta Products Corporation, Research Triangle Park, NC 27709 USA (e-mail: yhu@ deltartp.com).

Digital Object Identifier 10.1109/TPEL.2008.2004558

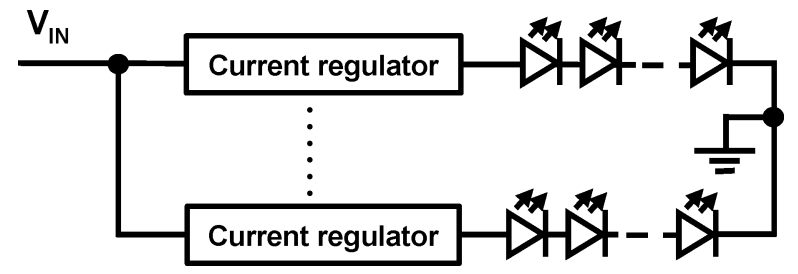

Fig. 1. LED driver with a current regulator in each string. Current regulator can be either linear or switch-mode type.

a below maximum voltage drop operate with reduced efficiencies. This drawback of the linear current regulator LED driver can be overcome by employing a more efficient switch-mode current regulator to drive each LED string [5]-[12]. Although this approach offers a higher operating efficiency compared to its linear counterpart, it has a higher component count and cost.

For LED drivers employing linear current regulators, the drive voltage is typically provided by a switch-mode preregulator [10], [13], as shown in Fig. 2. In the implementation shown in Fig. 2, the fixed output voltage of the preregulator is set by a voltage feedback at the level that ensures that current regulators for each string are capable of providing the desired current for the worst-case condition, i.e., for the maximum forward voltage drop of LED strings. In this LED driving approach, the only way to maintain high-efficiency operation of the linear regulators is to use LEDs with matched forward voltages, which inevitably increases the cost.

The efficiency of linear regulator LED drivers with a preregulator can be improved by sensing and regulating the minimum voltage of the linear regulators, as shown in Fig. 3. In this method, which was proposed in [14] and implemented with digital technology in [15], the lowest voltage drop across the linear regulators $\left(V_{\mathrm{MIN}}\right)$ is detected through the sensing diodes and compared with reference voltage $V_{\mathrm{REF}}$ so that the output voltage of the preregulator is automatically adjusted to keep the minimum linear regulator voltage to $V_{\mathrm{MIN}}=V_{\mathrm{REF}}-V_{\mathrm{F}}$, where $V_{\mathrm{F}}$ is the forward voltage drop of the sensing diodes. The efficiency performance of this approach is strongly affected by the selection of reference voltage $V_{\mathrm{REF}}$ and characteristics of the sensing diodes, primarily their temperature dependence. Because of the tolerances of minimum voltage drops of linear current regulators, i.e., tolerances of dropout voltages, reference voltage $V_{\mathrm{REF}}$ must be selected above the anticipated worst-case voltage, which is the highest dropout voltage expected. As a result of an increased reference voltage required to provide the worst-case design margin, the efficiency of the driver in Fig. 3 is always lower than the possible maximum efficiency. Moreover, because of the strong temperature dependence of the forward voltage of the sensing diodes, the actual sensed minimum 


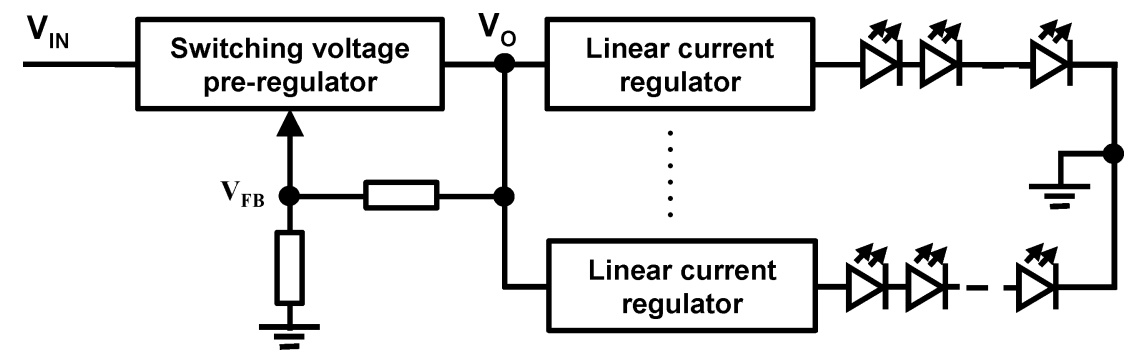

Fig. 2. LED driver with a linear current regulator in each string and switch-mode preregulator.

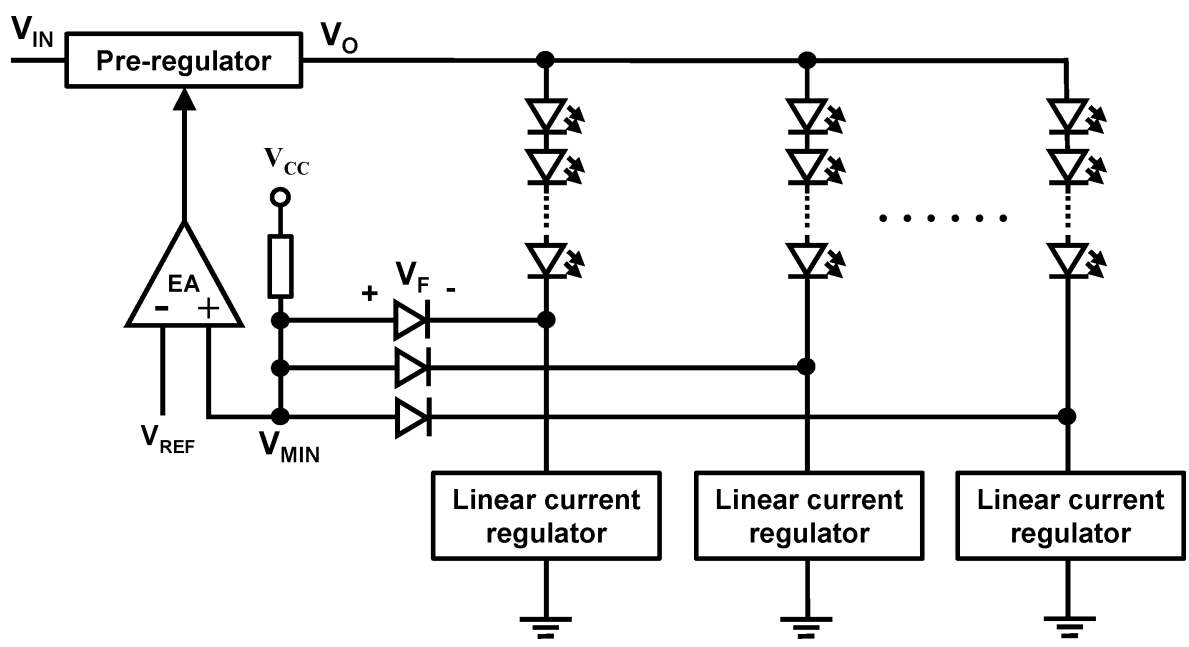

Fig. 3. LED driver with a switching voltage preregulator and detection circuit for minimum voltage drop of linear regulators.

voltage drop across the linear regulators varies with the operating temperature, causing a significant variation of the power loss. The detrimental effects of the nonoptimal reference voltage $V_{\mathrm{REF}}$ and temperature dependence of the forward voltage drop of the sensing diodes are progressively more pronounced as the number of LEDs in a string decreases.

In this paper, a novel linear regulator LED driver for paralleled LED strings that employs a voltage preregulator with adaptive output voltage and that offers the maximum efficiency is introduced. In this LED driver, efficiency maximization is achieved by eliminating the sensing of the voltage drops across the linear regulators, i.e., by removing the external voltage feedback for the adjustment of the output voltage of the preregulator.

\section{Proposed LED Driver With Adaptive Drive Voltage}

The proposed LED driver shown in Fig. 4 consists of a preregulator and multiple linear current regulators connected in series with the LED strings. Current $I_{\mathrm{L} i}(i=1,2, \ldots, n)$ through each LED string is regulated by a corresponding linear current regulator by sensing the string current with sensing resistor $R_{\mathrm{S} i}$ and by comparing the sensed voltage with reference $V_{\operatorname{REF} i}$. The error between the sensed and desired current in each string is then processed by corresponding error amplifier $\mathrm{EA}_{i}$ so that erroramplifier output voltage $V_{\mathrm{EA} i}$, which is also the gate-to-source $V_{\mathrm{GS} i}$ of regulating MOSFET device $Q_{i}$, is adjusted to a level necessary to maintain the desired string current $V_{\mathrm{REF} i} / R_{\mathrm{S} i}$.

In the proposed circuit in Fig. 4, the outputs of all error amplifiers are OR-ed via diodes $D_{1}$ through $D_{n}$ to detect maximum error-amplifier output voltage $V_{\text {EAMAX }}$, which corresponds to the LED string with the highest voltage drop. Voltage $V_{\mathrm{E}}$ at the cathodes of the OR-ing diodes, which is equal to the difference between $V_{\text {EAMAX }}$ and forward voltage drop $V_{\mathrm{F}}$ of the detecting diode, is then applied to the input of the control circuit of the preregulator to adjust the output voltage to an optimal level. For a switch-mode preregulator, the control circuit in Fig. 4 is a pulsewidth modulator (PWM) that converts the control voltage $V_{\mathrm{E}}$ to duty cycle $D$. This modulator is usually implemented inside the preregulator as a part of the control IC.

Generally, the switching preregulator can be implemented using any nonisolated or isolated topology, depending on the inputvoltage range and the number of LEDs in a string. Typically, LED drivers employ the boost, buck, or buck/boost topologies.

In the proposed LED driver, which does not use any sensing of the voltage across the linear regulators, a proper adjustment of the output voltage of the preregulator is achieved by exploiting a relatively strong dependence between drain-to-source voltage $V_{\mathrm{DS}}$ and gate-to-source voltage $V_{\mathrm{GS}}$ of a MOSFET operating in the linear (ohmic) region. Namely, in the linear region, i.e., when $V_{\mathrm{DS}}<V_{\mathrm{GS}}-V_{\mathrm{TH}}$, drain-to-source current $I_{\mathrm{DS}}$ is given by

$$
I_{\mathrm{DS}}=C_{\mathrm{FET}}\left(V_{\mathrm{GS}}-V_{\mathrm{TH}}-\frac{V_{\mathrm{DS}}}{2}\right) V_{\mathrm{DS}}
$$

where $C_{\mathrm{FET}}$ is a constant (in amperes per square volt) and $V_{\mathrm{TH}}$ is the turn-on threshold of the MOSFET [17].

As can be seen from Fig. 5, which shows a plot of (1) for a given drain-to-source current $I_{\mathrm{DS}}$, drain-to-source voltage 


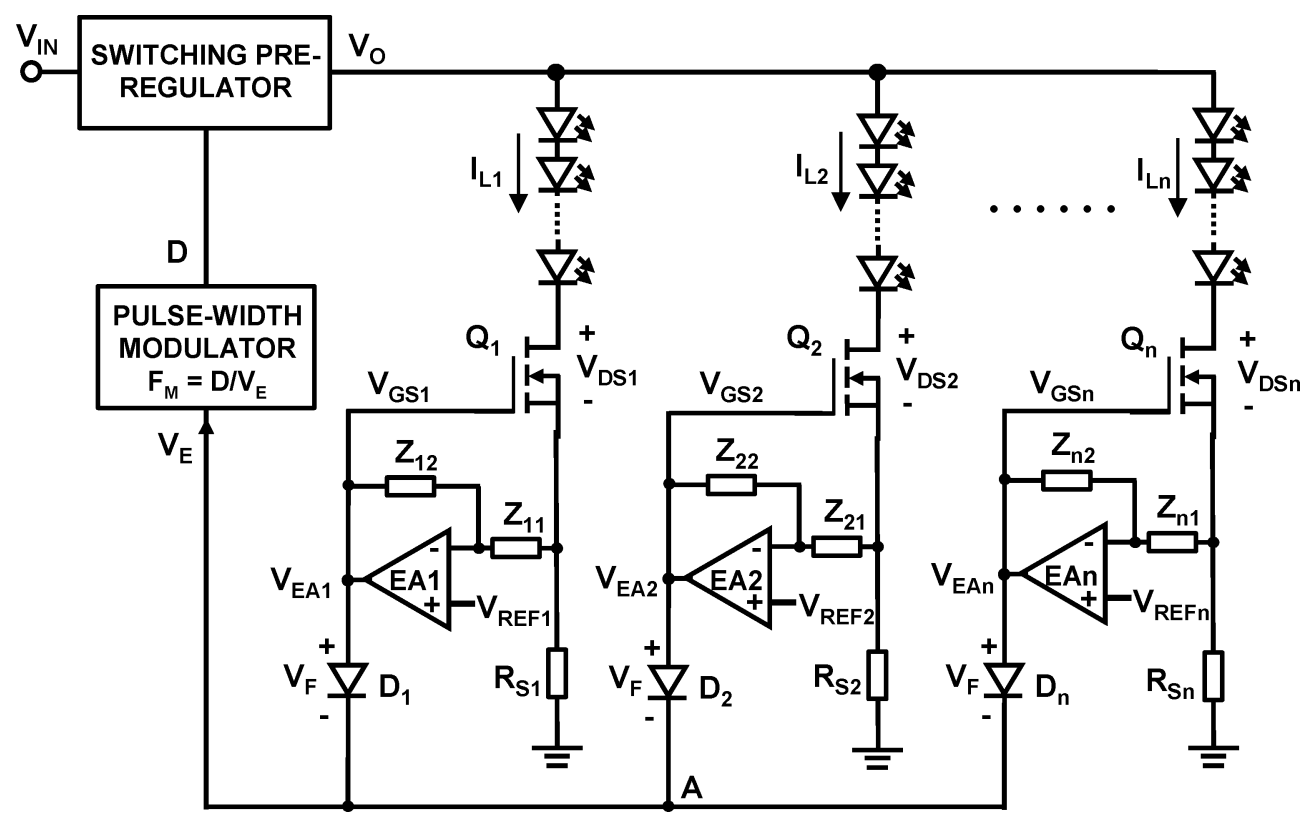

Fig. 4. Proposed LED driver with adaptive drive voltage for linear current regulators.

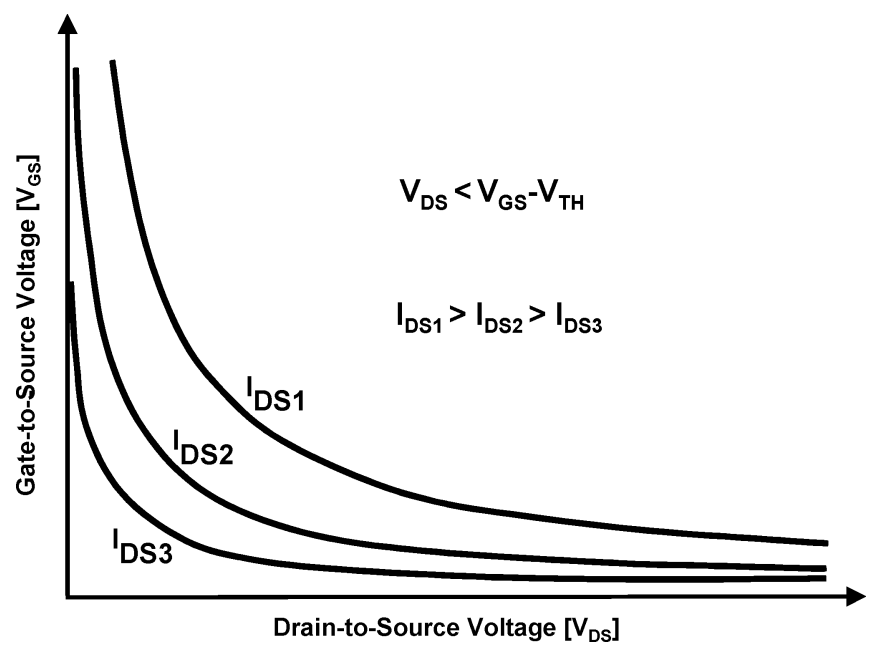

Fig. 5. Linear-region dependence of gate-to-source voltage on drain-to-source voltage of MOSFET for different drain-to-source currents.

$V_{\mathrm{DS}}$ decreases as gate-to-source voltage $V_{\mathrm{GS}}$ increases and vice versa. This dependence is exactly what is required in the LED driver in Fig. 4 to properly adjust the output voltage of the preregulator so that the maximum LED driver efficiency is achieved.

This self-adjusting feature of the proposed circuit can be further understood by considering its behavior in the presence of various disturbances. For example, if the voltage of the LED string with the highest voltage drop that controls the output voltage of the preregulator is increased, voltage $V_{\mathrm{DS}}$ of the corresponding current-regulating MOSFET that already has the lowest $V_{\mathrm{DS}}$ will decrease, causing a decrease in its drain-to-source current $I_{\mathrm{DS}}$. As a result, the error between reference voltage $V_{\mathrm{REF} i}$ and current-sensing-resistor voltage will increase, causing the output of the error amplifier, which is also the gate-tosource voltage $V_{\mathrm{GS}}$, to increase to maintain the desired LED current. At the same time, the increased error-amplifier voltage, which is also applied to the input of the modulator, increases the duty cycle of the preregulator so that the preregulator output voltage is also increased to compensate for the LED voltage change. Using a similar analysis, it can also be shown that the proposed driver rejects drive-voltage changes caused by variations in the preregulator input voltage.

It should be noted that in this sensorless, adaptive drivevoltage method, the drive voltage is always self-adjusted to the minimum voltage required to maintain the desired current through the LED string with the maximum voltage drop. As a result, all linear current regulators in the proposed LED driver operate with minimized voltage drops, which makes the efficiency of the driver maximal.

The brightness of each LED string powered by the proposed driver circuit can be individually controlled by adjusting the average LED string current via PWM dimming, as shown in Fig. 6. The frequency of the PWM dimming control signal is typically in the 100-400 Hz range. In the driver circuit in Fig. 6, when the PWM control signal is high, switch $\mathrm{QD}_{1}, \mathrm{QD}_{2}, \ldots, \mathrm{QD}_{n}$ is turned on, the anode of $\mathrm{DD}_{1}, \mathrm{DD}_{2}, \ldots, \mathrm{DD}_{n}$ is pulled low, and the diode becomes reverse-biased, and the LEDs are lit with the preset current. However, when the PWM control signal is low, switch $\mathrm{QD}_{1}, \mathrm{QD}_{2}, \ldots, \mathrm{QD}_{n}$ is turned off, and diode $\mathrm{DD}_{1}, \mathrm{DD}_{2}, \ldots, \mathrm{DD}_{n}$ conducts. By properly designing the voltage divider consisting of resistors $R_{i 1}$ and $R_{i 3}(i=1, \ldots, n)$ so that the voltage across resistor $R_{i 1}$, i.e., $R_{i 1}\left(V_{\mathrm{CC}}-V_{\mathrm{F}}\right) /\left(R_{i 1}+R_{i 3}\right)$, is greater than reference voltage $V_{\mathrm{REF} i}\left(V_{\mathrm{F}}\right.$ is the forward voltage drop of diodes $\mathrm{DD}_{i}$ ), the output of error amplifier $\mathrm{EA}_{i}$ becomes low when $\mathrm{PWM}_{i}$ is low, turning off the current regulating transistor $Q_{i}$ and the corresponding LED string. The LED string with the next highest voltage drop then sets control voltage $V_{\mathrm{E}}$. In the case where all PWM dimming signals are simultaneously low, diodes $D_{1}$ to $D_{n}$ are all reverse-biased, and control voltage $V_{\mathrm{E}}$ decreases to zero, preventing the preregulator from continuing to charge the output capacitor when all the LED strings are turned off. 


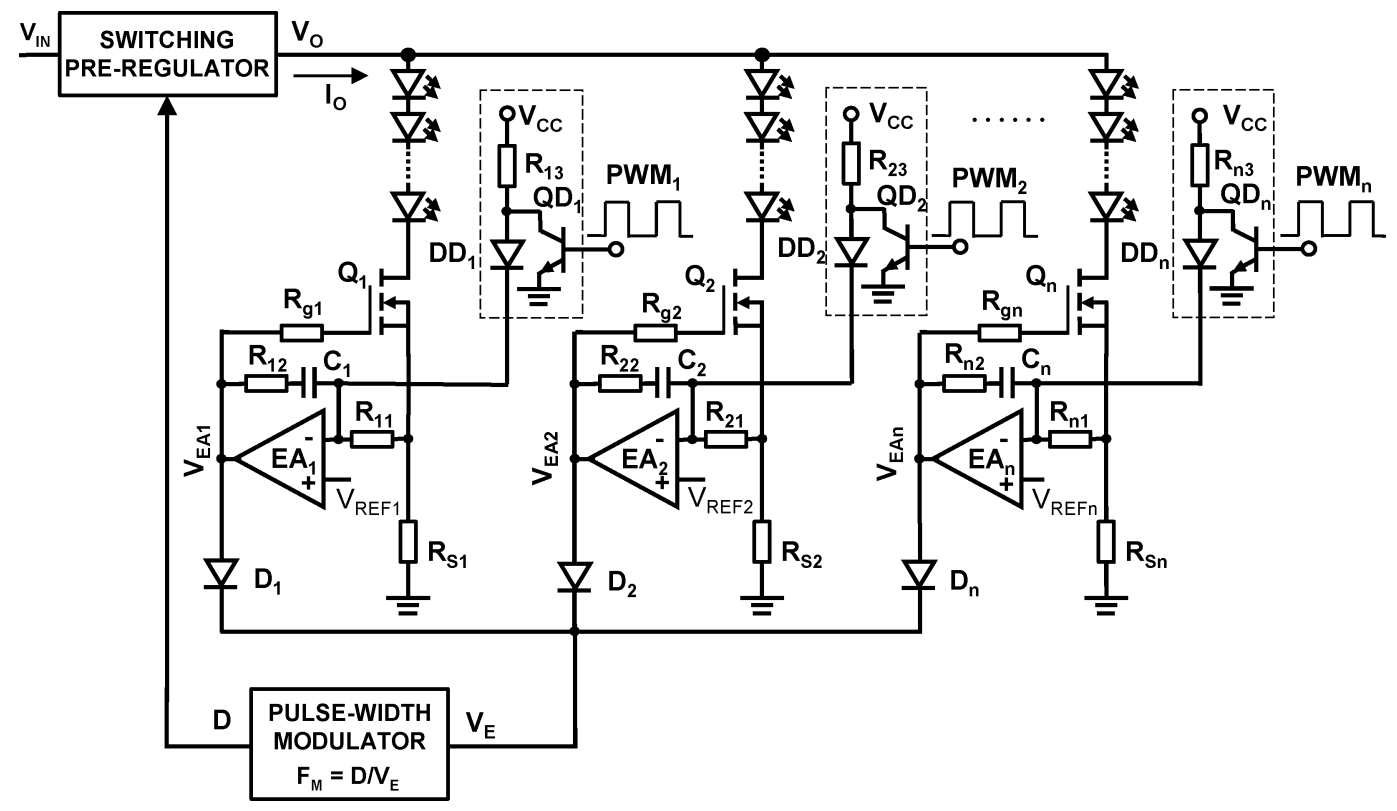

Fig. 6. LED driver with individual PWM dimming of LED strings.

The major drawback of PWM dimming is that if all the LED strings are turned on or off simultaneously, the input-output power periodically undergoes abrupt changes, causing large pulsating input/output current, degraded EMI performance, decreased operating efficiency, and increased power bus ripple. In order to alleviate this problem, sequential PWM dimming can be applied, as described in [15] and [16]. This type of dimming employs phased PWM dimming control signals to adjust LED brightness, which means that different LED strings draw the power consecutively by a certain phase delay rather than simultaneously. By incorporating sequential PWM dimming into the proposed LED driver, not only can the instantaneous output current drawn from the preregulator be reduced, but also output voltage $V_{\mathrm{O}}$ of the preregulator is self-adjusted to a voltage that results in a maximum operating efficiency. Fig. 7(a) and (b) shows predicted waveforms of the output current and voltage waveforms of the preregulator for the proposed LED driver with three sequentially PWM dimmed LED strings. The phase delay between two consecutive PWM dimming signals is $120^{\circ}$. When the PWM dimming duty cycle is less than $1 / 3$, as shown in Fig. 7(a), only one LED string is turned on at any time, and the output voltage of the preregulator tracks the LED-string voltage in a step fashion with three levels, resulting in a minimum voltage drop across the corresponding linear current regulator each time it is enabled. When the PWM dimming duty cycle is greater than 1/3, as shown in Fig. 7(b), there exists overlapped turn-on time between LED strings, and the output voltage of the preregulator always tracks the maximum of LED string voltages. Generally, for a driver with $n$ LED strings, there is always overlapped turn-on time between LED strings if the PWM duty cycle is greater than $1 / n$. It is noted that as the PWM dimming duty cycle goes lower, the benefit of sequential PWM dimming is more prominent since the overlapped time is shorter and each LED string is driven with a more optimized drive voltage.

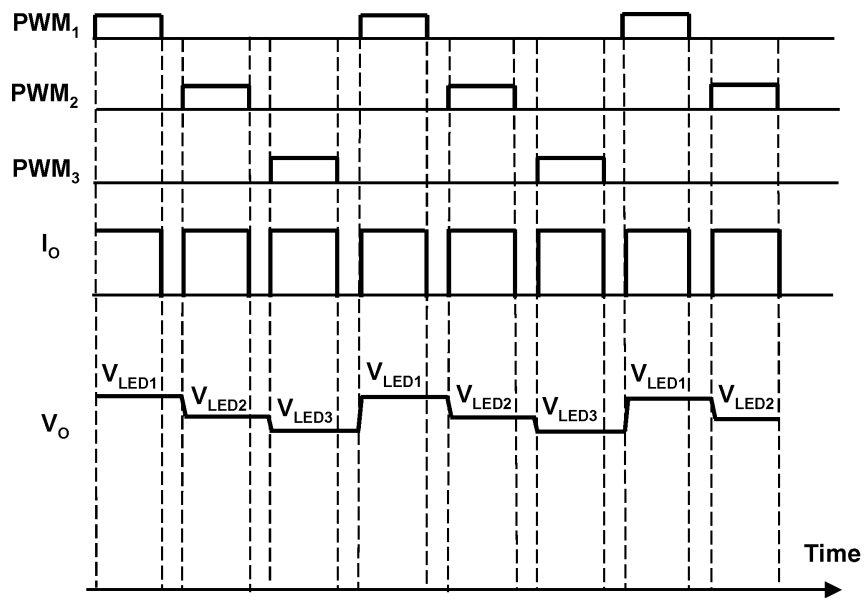

(a)

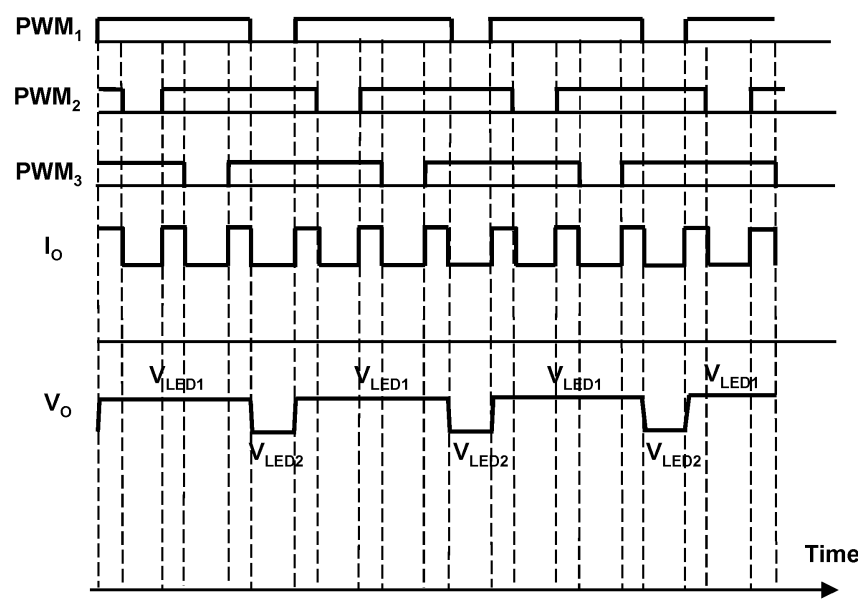

(b)

Fig. 7. Output current and voltage waveforms of the preregulator with three sequentially PWM dimmed LED strings assuming $V_{\mathrm{LED} 1}>V_{\mathrm{LED} 2}>V_{\mathrm{LED}}$. (a) Dimming duty cycle $<1 / 3$. (b) Dimming duty cycle $>1 / 3$. 


\section{DESIGN CONSIDERATIONS}

To achieve the optimal performance of the proposed LED driver, i.e., to minimize the power loss of the linear regulators, a critical design step is to determine the required gain of the pulsewidth modulator of the preregulator because the modulator gain determines the regulation performance of the voltage loop that self-adjusts the LED drive voltage. Of course, the performance optimization of the switching preregulator power stage and the linear current regulators is still crucial for the overall driver performance. However, since the optimization methods for switch-mode and linear regulators are well known and understood, they will not be addressed in this paper.

Generally, to ensure that the proposed LED driver works properly, i.e., its linear current regulators operate with a minimum voltage drop, the output voltage of the preregulator has to be designed with a proper output-voltage adjustment range. This voltage adjustment range is determined by the number of LEDs in a string and the forward-voltage-drop range of the LEDs. Typical forward-voltage-drop $V_{\mathrm{FLED}}$ of a red color LED is between 2.5 and $3.5 \mathrm{~V}$ at $350 \mathrm{~mA}$. For a driver intended to be used for driving LED strings with a high number, as well as a low number of LEDs, including a single LED string, the output voltage range of the preregulator has to be relatively wide. Specifically, for a driver that can drive strings that have between 1 and 10 LEDs, the preregulator must be able to provide a voltage adjustment in approximately $2-40 \mathrm{~V}$ range. Generally, to achieve such a wide voltage adjustment range, the duty cycle of the preregulator must be able to vary in a wide range too. For example, for a buck/boost type preregulator operating from a $12-\mathrm{V}$ input, the output voltage variation from 2 to $40 \mathrm{~V}$ requires a duty cycle variation from $14 \%$ to $77 \%$.

Since in the proposed LED driver the pulsewidth modulator is driven by the output of the error amplifier with the highest voltage, and since this voltage is only a diode-voltage drop lower than the gate-to-source voltage of the corresponding current regulating MOSFET, modulator input voltage $V_{\mathrm{E}}$ is relatively high. Typically, it is in the 5-15 V range. As a result, modulator gain $F_{\mathrm{M}}=D / V_{\mathrm{E}}$ is relatively low. For example, for gate-to-source voltage $V_{\mathrm{GS}}=10 \mathrm{~V}$, modulator gain $F_{\mathrm{M}}$ that can provide a duty cycle in the $14 \%-77 \%$ range must be lower than 0.014 because $F_{\mathrm{M}}=0.14 / 10=0.014$. An implementation of the modulator with such a low gain may not be practical. In addition, a low modulator gain also has a detrimental effect on the performance of the driver, as will be discussed later.

A more accurate relationship between modulator gain $F_{\mathrm{M}}$, input voltage of the preregulator $V_{\mathrm{IN}}$, LED current $I_{\mathrm{LED}}$, and voltage drop across the LED string $V_{\mathrm{LED}}$ can be derived by recognizing that in the proposed circuit in Fig. 4, the output voltage of the preregulator is approximately equal to the voltage drop of the LED string with maximum voltage drop, i.e.,

$$
V_{\mathrm{O}}=V_{\mathrm{LED}}+V_{\mathrm{DS}}+V_{\mathrm{RS}} \approx V_{\mathrm{LED}}
$$

because for linear current regulators with the current-regulating MOSFET operating in the linear region $V_{\mathrm{DS}} \ll V_{\mathrm{LED}}$, whereas current-sensing resistor value $R_{\mathrm{S}}$ can always be selected so that $V_{\mathrm{RS}}=R_{\mathrm{S}} I_{\mathrm{LED}} \ll V_{\mathrm{LED}}$.
Assuming a CCM buck/boost-type preregulator, i.e.,

$$
V_{\mathrm{O}}=\frac{D}{1-D} V_{\mathrm{IN}}
$$

and using modulator input-to-output relationship

$$
D=F_{\mathrm{M}}\left(V_{\mathrm{GS}}-V_{\mathrm{F}}\right)
$$

to eliminate $D$ from (4), the gate-to-source voltage that is required to provide desired output voltage can be obtained as

$$
V_{\mathrm{GS}}=\frac{V_{\mathrm{LED}}}{V_{\mathrm{LED}}+V_{\mathrm{IN}}} \frac{1}{F_{\mathrm{M}}}+V_{\mathrm{F}} .
$$

Since the range of gate-to-source voltage $V_{\mathrm{GS}}$ is constrained between a minimum value $V_{\mathrm{GSMIN}}$, which is above the threshold voltage $V_{\mathrm{TH}}$, and maximum value $V_{\mathrm{GSMAX}}$, which is below the gate-to-source breakdown voltage, the range of modulator gain $F_{\mathrm{M}}$ is also constrained. From (5), the modulator gain range can be calculated as

$$
\begin{aligned}
\frac{V_{\mathrm{LED}}}{V_{\mathrm{LED}}+V_{\mathrm{IN}}} \frac{1}{V_{\mathrm{GSMAX}}-V_{\mathrm{F}}} & <F_{\mathrm{M}} \\
< & \frac{V_{\mathrm{LED}}}{V_{\mathrm{LED}}+V_{\mathrm{IN}}} \frac{1}{V_{\mathrm{GSMIN}}-V_{\mathrm{F}}} .
\end{aligned}
$$

Minimum gate-to-source voltage $V_{\mathrm{GSMIN}}$ required to provide desired LED current $I_{\mathrm{LED}}$ and at the same time maintain the MOSFET operation in the linear region can be calculated from (1) by recognizing that at the boundary of the linear and saturation regions, $V_{\mathrm{DS}}=V_{\mathrm{GS}}-V_{\mathrm{TH}}$ so that relationship (1) can be written as

$$
\begin{gathered}
I_{\mathrm{DS}}=\frac{1}{2} C_{\mathrm{FET}}\left(V_{\mathrm{GS}}-V_{\mathrm{TH}}\right)^{2} \\
V_{\mathrm{GSMIN}}=\sqrt{\frac{2 I_{\mathrm{LED}}}{C_{\mathrm{FET}}}}+V_{\mathrm{TH}}
\end{gathered}
$$

and

$$
F_{\mathrm{M}} \leq \frac{V_{\mathrm{LED}}}{V_{\mathrm{LED}}+V_{\mathrm{IN}}} \frac{1}{\sqrt{\left(2 I_{\mathrm{LED}} / C_{\mathrm{FET}}\right)}+V_{\mathrm{TH}}-V_{\mathrm{F}}} .
$$

Relationship (6) is shown in Fig. 8 as a plot of an allowable modulator gain range as a function of LED-string voltage $V_{\mathrm{LED}}$ for a current-regulating MOSFET employing IRF540 device $\left(V_{\mathrm{TH}}=2.90 \mathrm{~V}, C_{\mathrm{FET}}=6.41 \mathrm{~A} / \mathrm{V}^{2}\right)$ and operating in the linear region with $I_{\mathrm{DS}}=I_{\mathrm{LED}}=0.7 \mathrm{~A}$, assuming input voltage $V_{\mathrm{IN}}=24 \mathrm{~V}$, and OR-ing diode voltage drop $V_{\mathrm{F}}=0.7 \mathrm{~V}$. In Fig. 8, any modulator gain above $F_{\mathrm{MMAX}}$ will lead to an undesirable operation in the saturation region of the MOSFET, whereas for a modulator gain below $F_{\mathrm{MMIN}}$, the maximum desirable gate-to-source voltage will be exceeded. For example, for a string voltage in the range from 56 to $80 \mathrm{~V}$, which corresponds to a string of 16 white LEDs, a modulator gain of around 0.2 can be chosen. However, for a string voltage range of 3.5-5 V that corresponds to one LED, the modulator gain should be kept below 0.05 , i.e., around 0.04 .

The selection of a lower modulator gain results in a lower power loss in the linear current regulators because a lower modulator gain requires a higher gate-to-source voltage $V_{\mathrm{GS}}$, 


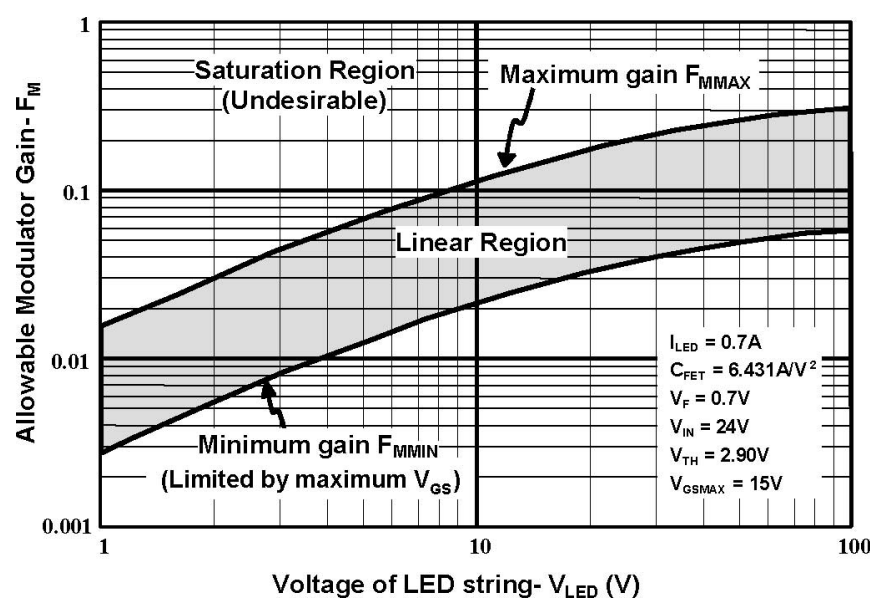

Fig. 8. Plot of allowable range of modulator gain $F_{\mathrm{M}}$ as a function of LED string voltage.

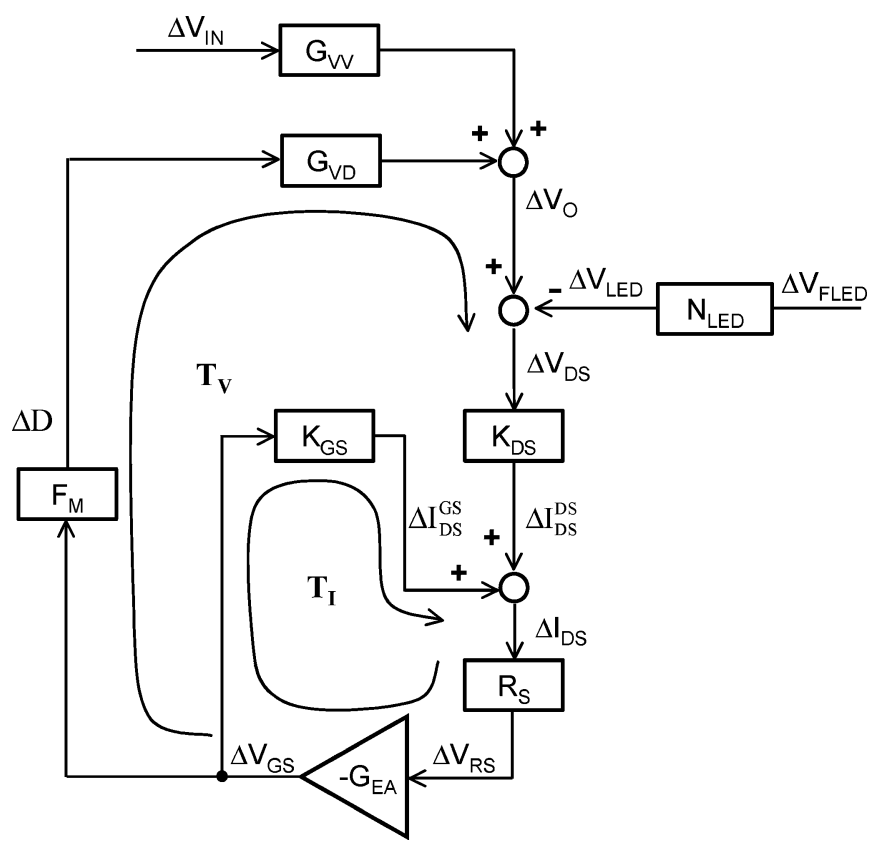

Fig. 9. Small-signal block diagram of the proposed driver.

and therefore, reduces drain-to-source voltage $V_{\mathrm{DS}}$. A very low modulator gain is not desirable due to several reasons. First, the requirement for a modulator gain below 0.2 excludes the use of internal modulators that are an integral part of today's PWM control ICs since the modulator gain of the majority of these controller ICs is greater than 0.2. Second, the implementation of a discrete modulator with a very low gain may not be practical. Namely, because the gain of the commonly employed sawtooth modulator is given by $F_{\mathrm{M}}=1 / V_{\mathrm{RAMP}}$, where $V_{\mathrm{RAMP}}$ is the height of the ramp [18], a modulator gain of 0.04 requires a ramp of $25 \mathrm{~V}$, which may not be practical in a LED driver that operates from an input voltage of $24 \mathrm{~V}$ or below. In addition, a low modulator gain has a detrimental effect on the voltage self-adjustment performance because it reduces the loop gain of the voltage loop.

To examine the effect of the modulator gain on the voltageloop regulation performance, Fig. 9 shows a small-signal block diagram of the proposed driver. In Fig. $9, K_{\mathrm{DS}}$ and $K_{\mathrm{GS}}$ are small-signal gains of the MOSFET operating in the linear region defined as

$$
K_{\mathrm{DS}}=\left.\frac{\Delta I_{\mathrm{DS}}}{\Delta V_{\mathrm{DS}}}\right|_{\Delta V_{\mathrm{GS}}=0}
$$

and

$$
K_{\mathrm{GS}}=\left.\frac{\Delta I_{\mathrm{DS}}}{\Delta V_{\mathrm{GS}}}\right|_{\Delta V_{\mathrm{DS}}=0} .
$$

Using relationship (1), $K_{\mathrm{DS}}$ and $K_{\mathrm{GS}}$ can be derived as

$$
K_{\mathrm{DS}}=C_{\mathrm{FET}}\left(V_{\mathrm{GS}}-V_{\mathrm{TH}}-V_{\mathrm{DS}}\right)
$$

and

$$
K_{\mathrm{GS}}=C_{\mathrm{FET}} V_{\mathrm{DS}} .
$$

Blocks $G_{\mathrm{VD}}$ and $G_{\mathrm{VV}}$ in Fig. 9 represent the control-tooutput and audio-susceptibility transfer function of the switching preregulator, respectively [18]. For a buck/boost converter operating in CCM, these two transfer functions at low frequencies are given by

$$
G_{\mathrm{VD}}=\left.\frac{\Delta V_{\mathrm{O}}}{\Delta D}\right|_{\Delta V_{\mathrm{IN}}=0} ^{=} \frac{V_{\mathrm{IN}}}{(1-D)^{2}}
$$

and

$$
G_{\mathrm{VV}}=\left.\frac{\Delta V_{\mathrm{O}}}{\Delta V_{\mathrm{IN}}}\right|_{\Delta D=0} ^{=} \frac{D}{1-D} .
$$

From Fig. 9, current loop gain $T_{\mathrm{I}}$ and voltage loop gain $T_{\mathrm{V}}$ are defined as

$$
T_{\mathrm{I}}=G_{\mathrm{EA}} K_{\mathrm{GS}} R_{\mathrm{S}}
$$

and

$$
T_{\mathrm{V}}=\frac{F_{\mathrm{M}} G_{\mathrm{VD}} K_{\mathrm{DS}} R_{\mathrm{S}} G_{\mathrm{EA}}}{1+T_{\mathrm{I}}}=\frac{F_{\mathrm{M}} G_{\mathrm{VD}} K_{\mathrm{DS}} R_{\mathrm{S}} G_{\mathrm{EA}}}{1+K_{\mathrm{GS}} R_{\mathrm{S}} G_{\mathrm{EA}}} .
$$

Since for a properly designed current regulation loop $T_{\mathrm{I}} \gg 1$, voltage loop gain can be expressed as

$$
T_{\mathrm{V}} \approx F_{\mathrm{M}} G_{\mathrm{VD}} \frac{K_{\mathrm{DS}}}{K_{\mathrm{GS}}} .
$$

Finally, voltage adjustment $\Delta V_{\mathrm{O}}$ of the proposed LED driver with respect to changes in the LED string voltage $\Delta V_{\mathrm{LED}}$ and input voltage $\Delta V_{\mathrm{IN}}$ are, respectively, given by

$$
\frac{\Delta V_{\mathrm{O}}}{\Delta V_{\mathrm{LED}}}=\frac{1}{\left(1 / T_{\mathrm{V}}\right)+1}
$$

and

$$
\frac{\Delta V_{\mathrm{O}}}{\Delta V_{\mathrm{IN}}}=\frac{G_{\mathrm{VV}}}{T_{\mathrm{V}}+1} .
$$

As can be seen from (18), for a given power stage and linear current regulator, the voltage-loop gain can only be adjusted by the selection of modulator gain $F_{\mathrm{M}}$. For good loop regulation performance, $F_{\mathrm{M}}$ needs to be as high as possible. Namely, if modulator gain $F_{\mathrm{M}}$ is high enough so that voltage loop gain $T_{\mathrm{V}} \gg 1$, any change in LED-string voltage $V_{\mathrm{LED}}$ would be exactly compensated with a corresponding change of output voltage $V_{\mathrm{O}}$, i.e., the change of LED-string voltage $V_{\mathrm{LED}}$ would 


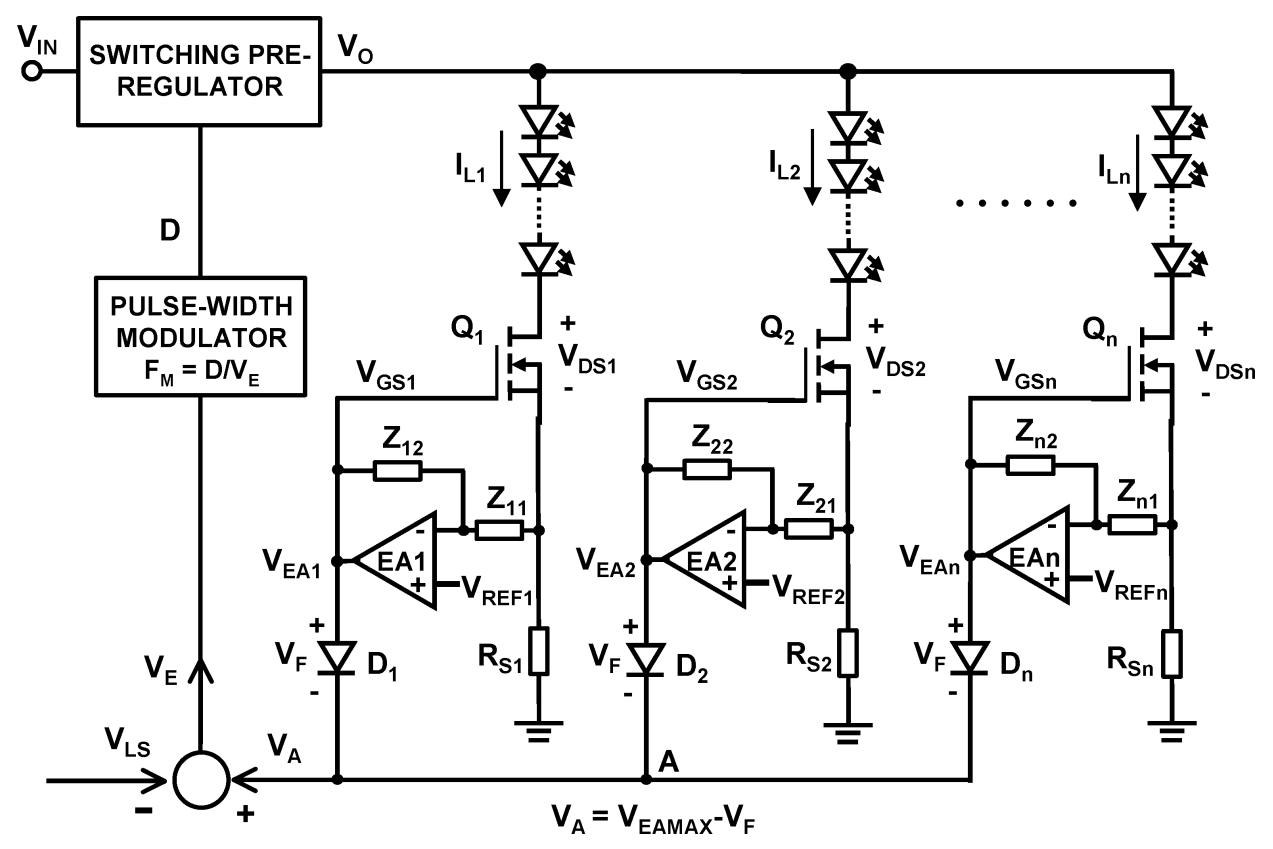

Fig. 10. Block diagram of the proposed LED driver with a level shifter.

not affect the efficiency performance of the driver. Furthermore, when $T_{\mathrm{V}} \gg 1$, according to (20), any change in input voltage $V_{\text {IN }}$ would result in essentially no change of the output voltage $V_{\mathrm{O}}$, i.e., the change of input voltage $V_{\mathrm{IN}}$ would also not affect the efficiency performance of the driver. However, in the proposed LED driver, voltage loop gain $T_{\mathrm{V}}$ is relatively low in order to achieve linear region operation of the MOSFET and maximize the efficiency, especially for LED strings with a low number of LEDs. For example, for $V_{\mathrm{LED}}=3.5 \mathrm{~V}, I_{\mathrm{LED}}=0.7 \mathrm{~A}, V_{\mathrm{IN}}=$ $24 \mathrm{~V}, D=0.127$, according to Fig. $8, F_{\mathrm{M}}$ needs to be below 0.05 . If $F_{\mathrm{M}}=0.04, V_{\mathrm{DS}}$ is $0.118 \mathrm{~V}$ and $V_{\mathrm{GS}}$ is $3.875 \mathrm{~V}$ so that voltage loop gain $T_{\mathrm{V}}=9.15$. With this voltage loop gain, a $1-\mathrm{V}$ decrease of the LED-string voltage would cause only a $0.90-\mathrm{V}$ decrease of output voltage, resulting in a $0.1-\mathrm{V}$ increase of the voltage drop and 70- $\mathrm{mW}$ (or 84.7\%) loss increase of the MOSFET. In addition, a 5-V change in the input voltage would cause a $71.66-\mathrm{mV}$ change in the drain-to-source voltage of the MOSFET, leading to $50-\mathrm{mW}$ (or $60.73 \%$ ) power loss increase.

The voltage loop performance of the proposed LED driver can be improved by employing a level shifter to reduce the voltage at the input of the modulator, as illustrated in Fig. 10. By subtracting a fixed voltage $V_{\mathrm{LS}}$ from voltage $V_{\mathrm{E}}$, the gain of the modulator can be increased without adversely affecting the current regulation or efficiency performance of the LED driver.

Following the same derivation procedure as for the case without voltage-level shifting, the maximum modulator range can be derived as

$$
\begin{gathered}
F_{\mathrm{M}} \leq \frac{V_{\mathrm{LED}} /\left(V_{\mathrm{LED}}+V_{\mathrm{IN}}\right)}{\sqrt{2\left(I_{\mathrm{LED}} / C_{\mathrm{FET}}\right)}+V_{\mathrm{TH}}-V_{\mathrm{F}}-V_{\mathrm{LS}}} \\
\left(V_{\mathrm{LS}}<V_{\mathrm{LSCRIT}}\right)
\end{gathered}
$$

where critical voltage shift level $V_{\text {LSCRIT }}$ is defined as

$$
V_{\mathrm{LSCRIT}}=\sqrt{2\left(I_{\mathrm{LED}} / C_{\mathrm{FET}}\right)}-V_{\mathrm{F}}+V_{\mathrm{TH}} .
$$

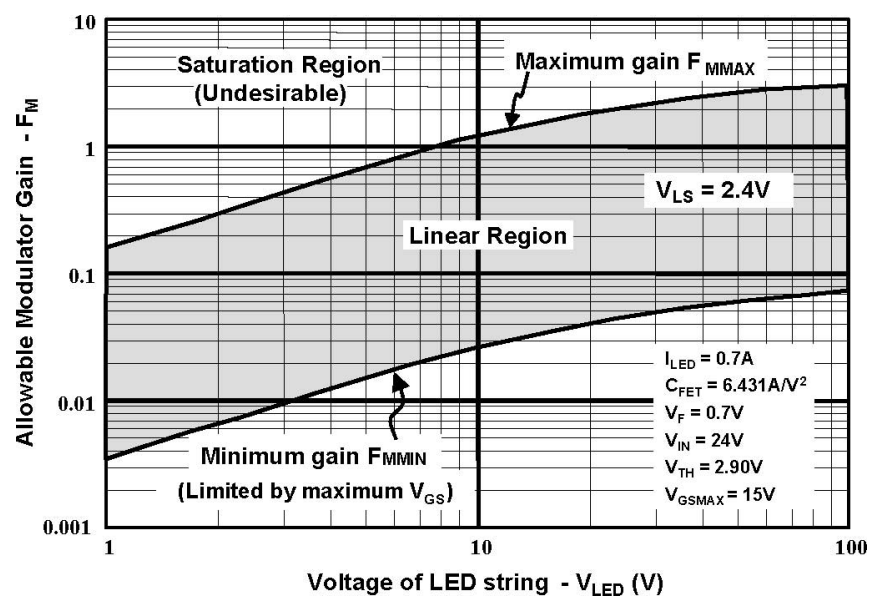

Fig. 11. Plot of the allowable range of modulator gain $F_{\mathrm{M}}$ as a function of LED string voltage $V_{\mathrm{LED}}$ with a $2.4-\mathrm{V}$ level shifter.

When $V_{\mathrm{LS}} \geq V_{\mathrm{LSCRIT}}, V_{\mathrm{GS}}$ is always greater than the required $V_{\mathrm{GSMIN}}$ to maintain the desired $I_{\mathrm{LED}}$, regardless of the modulator gain $F_{\mathrm{M}}$.

Minimum $F_{\mathrm{M}}$ that is constrained by the maximum gate-tosource breakdown voltage is given by

$$
F_{\mathrm{M}}>\frac{V_{\mathrm{LED}}}{V_{\mathrm{LED}}+V_{\mathrm{IN}}} \frac{1}{V_{\mathrm{GSMAX}}-V_{\mathrm{F}}-V_{\mathrm{LS}}} .
$$

Finally, the maximum voltage shift level for a given gain $F_{\mathrm{M}}$ is limited to

$$
V_{\mathrm{LS}} \leq V_{\mathrm{GSMAX}}-\frac{V_{\mathrm{LEDMIN}}}{V_{\mathrm{LEDMIN}}+V_{\mathrm{IN}}} \frac{1}{F_{\mathrm{M}}}-V_{\mathrm{F}}
$$

where $V_{\text {LEDMIN }}$ is the minimum LED-string voltage. 


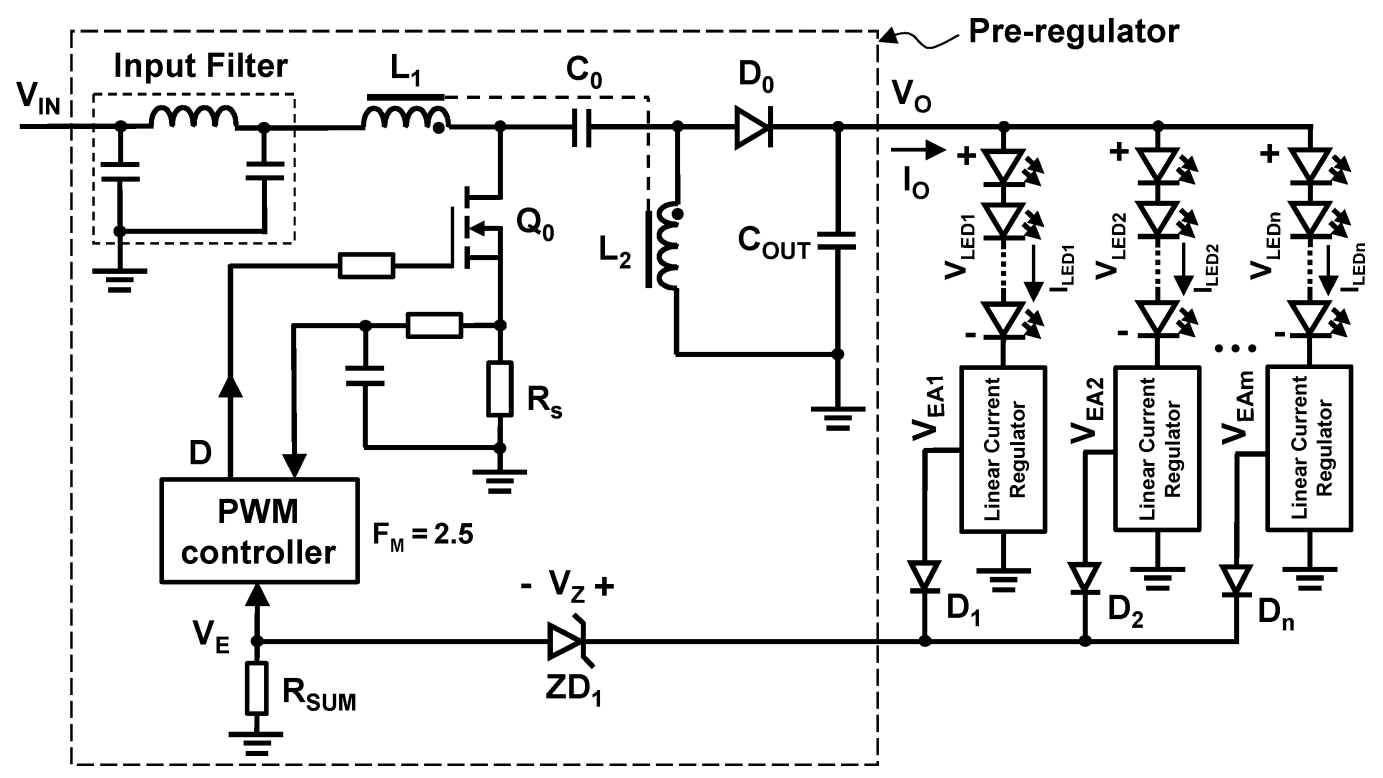

Fig. 12. Experimental LED driver with adaptively controlled drive voltage employing SEPIC preregulator.

Fig. 11 shows the plot of allowable range of modulator gain $F_{\mathrm{M}}$ as a function of the LED-string voltage with a level shifter. Compared to the plot shown in Fig. 8 for the case without a level shifter, the maximum gain is increased significantly for the whole range of LED-string voltage under the same conditions. For example, with a $2.4-\mathrm{V}$ level shift and for a string voltage in the range from 56 to $80 \mathrm{~V}$, a modulator gain of around 2 can be chosen, whereas for a string voltage range of $3.5-5 \mathrm{~V}$, the modulator gain can be as high as 0.4 . These two gains are about ten times the gains for the case without a level shifter.

It should be noted that parameters $V_{\mathrm{TH}}$ and $C_{\mathrm{FET}}$ of the MOSFET and $V_{\mathrm{F}}$ of the diode vary with manufacture tolerance and operating temperature. Therefore, to ensure proper operation of the driver, the maximum modulator gain $F_{\mathrm{M}}$ must be determined from (9) or (21) based on the worst-case design, i.e., for the lowest of $C_{\mathrm{FET}}$, the highest of $V_{\mathrm{TH}}$, and the lowest of $V_{\mathrm{F}}$.

\section{Performance Evaluation}

The performance of the proposed LED driver was verified experimentally by building and testing an LED driver with a $120-\mathrm{kHz}$ single-ended primary inductor convertor (SEPIC) preregulator operating from a voltage source in $20-30 \mathrm{~V}$ range. The circuit diagram of the experimental circuit is shown in Fig. 12, whereas Table I lists its key components. The SEPIC topology was selected because it can work with an input voltage that is above or below its output voltage, which is advantageous for applications with an input voltage range overlapping the output voltage [19]-[21]. Four parallel strings of white LEDs (LXHL-LW3 C from Philips Lumileds), with eight LEDs series connected in each string, were used to evaluate the performance of the proposed LED driver.

The measured voltage drops of LED strings are: $V_{\mathrm{LED} 1}=$ $24.50 \mathrm{~V}, V_{\mathrm{LED} 2}=24.47 \mathrm{~V}, V_{\mathrm{LED} 3}=24.46 \mathrm{~V}$, and $V_{\mathrm{LED} 4}=$ $24.52 \mathrm{~V}$ at $350 \mathrm{~mA}$ with a LED current reference voltage of $210 \mathrm{mV}$. The current regulating MOSFET for the fourth LED string with the highest voltage drop has the highest gate-to-
TABLE I

KEY COMPONENT LIST

\begin{tabular}{|l|l|}
\hline & $\begin{array}{l}\text { Core: MS080125, } \mu_{\mathrm{i}}=125 \\
\mathrm{~L}_{1}, \mathrm{~L}_{2}\end{array}$ \\
& $\begin{array}{l}\text { Winding: AWG\# 23, } \mathrm{N}_{1}=\mathrm{N}_{2}=45 \mathrm{Ts} \\
\mathrm{L}_{1}=\mathrm{L}_{2}=148 \mu \mathrm{H}\end{array}$ \\
\hline $\mathrm{C}_{0}$ & Electrolytic, 220 uF, 35 V \\
\hline $\mathrm{D}_{0}$ & MBR2090CT, 90 V, 20 A \\
\hline $\mathrm{C}_{\mathrm{OuT}}$ & Electrolytic, $100 \mathrm{uF}, 100 \mathrm{~V}$ \\
\hline $\mathrm{Q}_{0}$ of pre-regulator & IRF540, 100 V, 28 A \\
$\mathrm{Q}_{1}-\mathrm{Q}_{4}$ of current regulators & \\
\hline ZD $\mathrm{D}_{1}$ & $1 \mathrm{~N} 4731, \mathrm{~V}_{\mathrm{Z}}=4.3 \mathrm{~V}$ \\
\hline $\mathrm{D}_{1}-\mathrm{D}_{4}$ & $1 \mathrm{~N} 4148,75 \mathrm{~V}$ \\
\hline PWM Controller & $\mathrm{UC3} 343$ \\
\hline Current Error Amplifiers & LM358 \\
\hline Switching frequency of SEPIC & $120 \mathrm{kHz}$ \\
\hline
\end{tabular}

source voltage, i.e., $V_{\mathrm{GS} 4}=6.28 \mathrm{~V}$ at $V_{\mathrm{IN}}=24 \mathrm{~V}$, and takes the feedback control of the output voltage of the preregulator. The other three gate-to-source voltages are $V_{\mathrm{GS} 1}=4.22 \mathrm{~V}, V_{\mathrm{GS} 2}=$ $3.91 \mathrm{~V}$, and $V_{\mathrm{GS} 3}=4.08 \mathrm{~V}$. The measured output voltage of the preregulator is $24.77 \mathrm{~V}$, resulting in low voltage drops of $V_{\mathrm{DS} 1}=60 \mathrm{mV}, V_{\mathrm{DS} 2}=90 \mathrm{mV}, V_{\mathrm{DS} 3}=100 \mathrm{mV}$, and $V_{\mathrm{DS} 4}=$ $40 \mathrm{mV}$. The efficiency of each linear current regulator and the overall efficiency of the proposed LED driver with adaptive drive voltage are shown in Figs. 13 and 14, respectively. As can be seen, compared with the conventional fixed-drive voltage control of the preregulator, the proposed adaptive control of drive voltage significantly reduces the power loss of the linear current regulators and improves the overall efficiency of the 


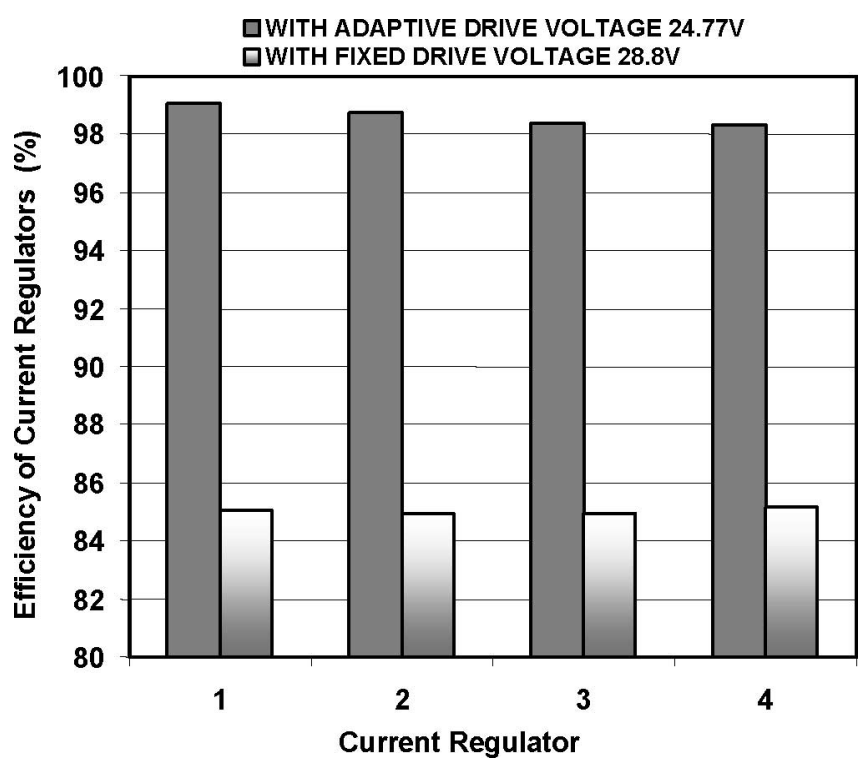

Fig. 13. Comparison of measured efficiency of current regulators with fixed and adaptive drive voltage.

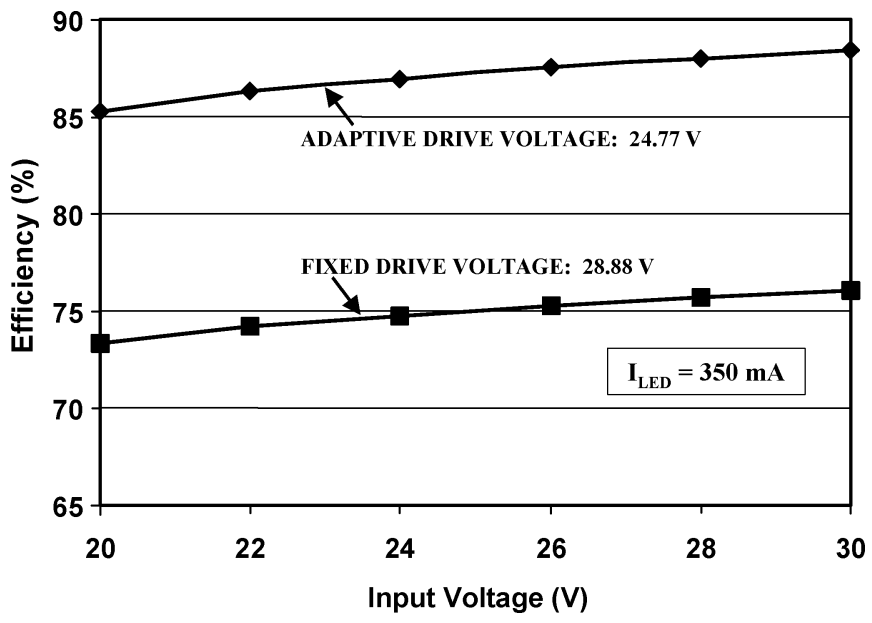

Fig. 14. Comparison of measured overall efficiency of LED driver with fixed and adaptive drive voltage as function of input voltage $V_{\mathrm{IN}}$.

LED driver dramatically. With a fixed-drive voltage control, the output voltage of the preregulator needs to be set based on the worst-case electrical specification of LEDs, i.e., at least $20 \%$ higher than the total typical voltage drop of all LEDs in one string. In this case, the typical total voltage drop of eight white LEDs is around $24 \mathrm{~V}$. To ensure current regulation of all LED strings, a drive voltage of $28.8 \mathrm{~V}$ has to be set, resulting in an about $15 \%$ efficiency loss in the linear current regulators. As a result, as shown in Fig. 14, the overall driver efficiency at $V_{\mathrm{IN}}=24 \mathrm{~V}$ is less than $75 \%$, which is $12 \%$ lower than that with the proposed adaptive drive voltage.

The performance of the proposed driver under sequential PWM dimming control was also verified experimentally. To demonstrate the ability of the proposed adaptive control to adjust the drive voltage, the mismatching of LED string voltages was exaggerated by inserting different-value resistors in the first, second, and third string. The measured voltage drops

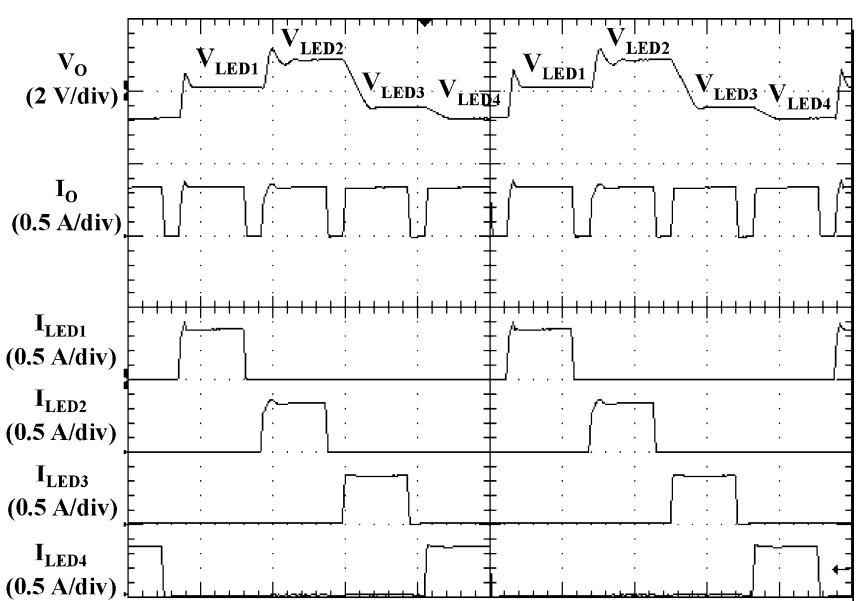

(a)

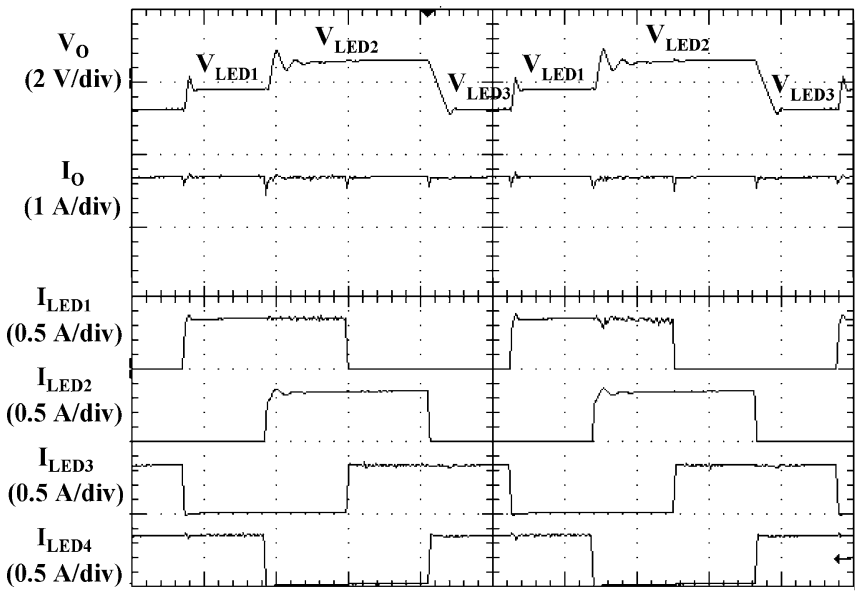

(b)

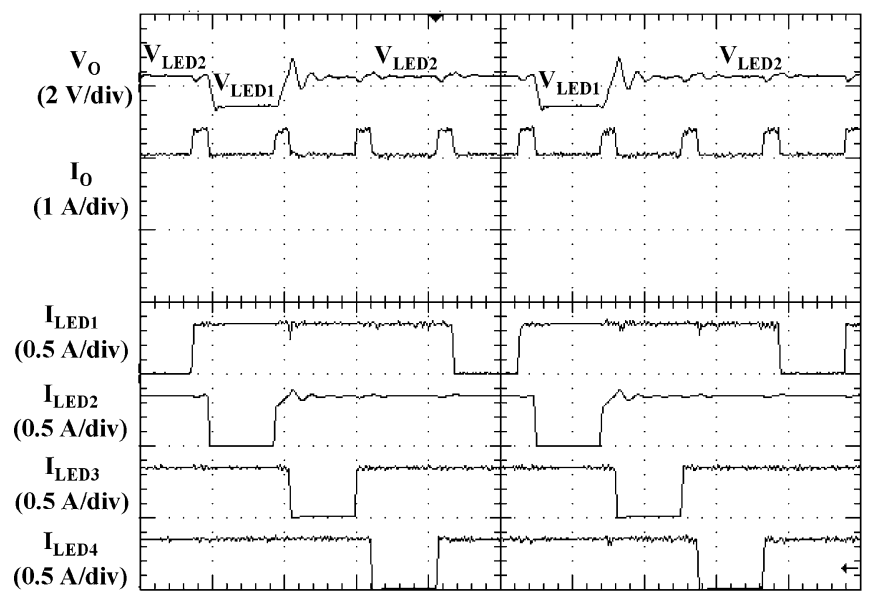

(c)

Fig. 15. Measured output voltage and current of preregulator, and current of each sequentially dimmed LED string of experimental LED driver. (a) Dimming duty cycle $=20 \%$. (b) Dimming duty cycle $=50 \%$. (c) Dimming duty cycle $=$ $80 \%$. Time base: $1 \mathrm{~ms} / \mathrm{div}$.

of the four strings were $V_{\mathrm{LED} 1}=25.37 \mathrm{~V}, V_{\mathrm{LED} 2}=26.26 \mathrm{~V}$, $V_{\mathrm{LED} 3}=24.84 \mathrm{~V}$, and $V_{\mathrm{LED} 4}=24.52 \mathrm{~V}$ at $0.35 \mathrm{~A}$. As shown in Fig. 15, the proposed driver adaptively adjusts the output voltage of the preregulator based on the actual maximum voltage of the loads. When the PWM dimming duty cycle is $20 \%$, the measured output voltage steps up or down between these four 
voltages, as shown in Fig. 15(a). The maximum output current of the preregulator is equal to the peak current of one LED string. When the PWM dimming duty cycle is $50 \%$, the measured output voltage has three levels, changing between $V_{\mathrm{LED} 1}$, $V_{\mathrm{LED} 2}$, and $V_{\mathrm{LED} 3}$, as shown in Fig. 15(b). In this case, it can be seen that although the load currents are pulsating, total output current $I_{O}$ drawn from the preregulator is constant. Fig. 15(c) further shows the measured waveforms when the PWM dimming duty cycle is $80 \%$. The drive voltage varies between $V_{\mathrm{LED} 1}$ and $V_{\mathrm{LED} 2}$, and the output current changes between 1.05 and $1.40 \mathrm{~A}\left(\Delta I_{\mathrm{O}}=0.35 \mathrm{~A}\right)$ instead of between 0 and $1.4 \mathrm{~A}$ $\left(\Delta I_{\mathrm{O}}=1.4 \mathrm{~A}\right)$, as in the case of nonsequential/simultaneous PWM dimming.

\section{CONCLUSION}

A LED driver with adaptive drive voltage has been proposed. The driver circuit consists of multiple linear current regulators and a voltage preregulator whose output voltage is automatically adjusted to a minimum voltage required to maintain the desired current through the linear regulators. Specifically, in the proposed driver, the voltage across the linear regulator in the LED string that exhibits highest voltage drop is always self-adjusted to the lowest voltage possible. As a result, the proposed LED driver exhibits significantly higher efficiency than its counterpart without the adjustment of the drive voltage. The minimization of the drive voltage on the proposed LED driver is achieved by sensing the gate-to-source voltage of the MOSFET-based linear current regulators and exploiting a relatively strong dependence between gate-to-source and drain-to-source voltage, instead of sensing the drain-to-source voltage and regulating the minimum voltage drop of the linear current regulators through a feedback control.

The performance of the proposed driver was experimentally verified on a four-string LED setup with eight white LEDs in each string. The $120-\mathrm{kHz}$ SEPIC topology powered from a $20-$ to $30-\mathrm{V}$ input source is used as the preregulator because the SEPIC topology can operate with the output voltage both below and above the input voltage. The measured improvement of the efficiency of the linear current regulators was approximately $15 \%$ compared to the corresponding implementation with a constant preregulator voltage. The proposed driver is particularly suitable for applications with sequential PWM dimming since its drive voltage is dynamically self-adjusted to a level that corresponds to minimum voltage drops across the linear current regulators, thus maximizing the conversion efficiency.

\section{REFERENCES}

[1] J. Y. Tsao, "Solid-state lighting: Lamps, chips, and materials for tomorrow," IEEE Circuits Devices Mag., vol. 20, no. 3, pp. 28-37, May/Jun. 2004.

[2] N. Narendran and Y. Gu, "Life of LED-based white light sources," $J$. Display Technol., vol. 1, no. 1, pp. 167-171, Sep. 2005.

[3] T. Komine and M. Nakagawa, "Fundamental analysis for visible-light communication system using LED lights," IEEE Trans. Consum. Electron., vol. 50, no. 1, pp. 100-107, Feb. 2004.

[4] G. Carraro, "Solving high-voltage off-line HB-LED constant-current control-circuit issues," in Proc. IEEE Appl. Power Electron. Conf. (APEC), 2007, pp. 1316-1318.
[5] H. Broeck, G. Sauerlander, and M. Vendt, "Power driver topologies and control schemes for LEDs," in Proc. IEEE Appl. Power Electron. Conf. (APEC), 2007, pp. 1319-1325.

[6] T. F. Pan, H. J. Chiu, S. J. Cheng, and S. Y. Chyng, "An improved singlestage flyback PFC converter for high-luminance lighting LED lamps," in Proc. 8th Int. Conf. Electron. Meas., Aug. 2007, vol. 4, pp. 212-215.

[7] H. J. Chiu and S. J. Cheng, "LED backlight driving system for large-scale LCD panels," IEEE Trans. Ind. Electron., vol. 54, no. 5, pp. 2751-2760, Oct. 2007.

[8] G. Spiazzi, S. Buso, and G. Meneghesso, "Analysis of a high-power-factor electronic ballast for high brightness light emitting diodes," in Proc. IEEE Power Electron. Spec. Conf. (PESC), Sep. 11-14, 2005, pp. 1494-1499.

[9] K. Zhou, J. G. Zhang, and S. Yuvarajan, "Quasi-active power factor correction circuit for HB LED driver," IEEE Trans. Power Electron., vol. 23 , no. 3, pp. 1410-1415, May 2008.

[10] S. Y. Tseng, S. C. Lin, and H. C. Lin, "LED backlight power system with auto-tuning regulation voltage for LCD panels," in Proc. IEEE Appl. Power Electron. Conf. (APEC), Feb. 2008, vol. 1, pp. 551-557.

[11] W. H. Chang, D. Chen, H. S. Nien, and C. H. Chen, "A digital boost converter to drive white LEDs," in Proc. IEEE Appl. Power Electron. Conf. (APEC), Feb. 2008, vol. 1, pp. 558-564.

[12] J. Sebastian, D. G. Lamar, M. Arias, M. Rodriguez, and M. M. Hernando, "A very simple control strategy for power factor correctors driving highbrightness lighting-emitting diodes," in Proc. IEEE Appl. Power Electron. Conf. (APEC), Feb. 2008, vol. 1, pp. 537-543.

[13] Y. H. Fan, C. J. Wu, C. C. Fan, K. W. Chih, and L. D. Liao, "A simplified LED converter design and implement," presented at the 9th Joint Conf. Inf. Sci. (JCIS), Taipei, Taiwan, Oct. 8-11 2006.

[14] L. Burgyan and F. Prinz, "High efficiency LED driver," U.S. Patent 6690 146, Feb. 10, 2004.

[15] M. Doshi and R. Zane, "Digital architecture for driving large LED arrays with dynamic bus voltage regulation and phase shifted PWM," in Proc. IEEE Appl. Power Electron. Conf. (APEC), 2007, pp. 287-293.

[16] C. C. Chen, C. Y. Wu, Y. M. Chen, and T. F. Wu, "Sequential color LED backlight driving system for LCD panels," IEEE Trans. Power Electron., vol. 22, no. 3, pp. 919-925, May 2007.

[17] S. M. Sze, Semiconductor Devices: Physics and Technology. New York: Wiley, 1985.

[18] R. W. Erickson and D. Maksimovic, Fundamentals of Power Electronics, 2nd ed. Boston, MA: Kluwer, 2001.

[19] D. Simonetti, J. Sebastián, and J. Uceda, "The discontinuous conduction mode SEPIC and Cuk power factor preregulators: Analysis and design," IEEE Trans. Ind. Electron., vol. 44, no. 5, pp. 630-637, Oct. 1997.

[20] J. Chen and C. Chang, "Analysis and design of SEPIC converter in boundary condition mode for universal-line power factor correction applications," in Proc. IEEE Power Electron. Spec. Conf. (PESC), 2001, pp. 742 747.

[21] Y. Q. Zhang and P. C. Sen, "A new soft-switching technique for buck, boost, and buck/boost converters," IEEE Trans. Ind. Appl., vol. 39, no. 6 , pp. 1775-1782, Nov./Dec. 2003

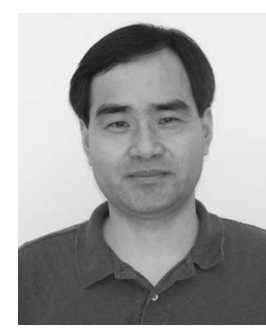

Yuequan Hu received the Bachelor's and Master's degrees in mechanical engineering and the Ph.D. degree in electrical engineering from the University of Science and Technology of China, Hefei, China, in 1989, 1992, and 1999, respectively.

He is currently a member of the R\&D Staff of Power Electronics Laboratory, Delta Products Corporation, Research Triangle Park, NC. His current research interests include high-intensity discharge (HID) lamp ballasts and LED drivers.

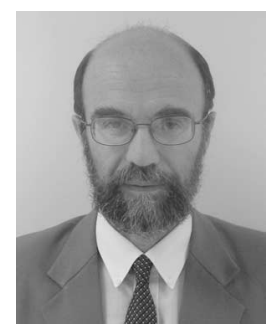

Milan M. Jovanović (F'01) received the Dipl.Ing. degree in electrical engineering from the University of Belgrade, Belgrade, Serbia.

$\mathrm{He}$ is currently the Chief Technology Officer of the Power Systems Business Group, Delta Electronics, Inc., Taipei, Taiwan. 